\title{
Efectos del Entrenamiento en Fortalezas Personales sobre los Síntomas Físicos de Adolescentes
}

\author{
Effects of Personal Strength Training on Adolescent Physical Symptoms
}

\author{
Juan Molina-Hernández y Ana García-León ${ }^{2}$
}

\begin{abstract}
Resumen
Se pretende comparar la eficacia de un programa de intervención centrado en el entrenamiento en fortalezas personales, con respecto a otro programa de intervención basado en el entrenamiento de habilidades sociales y técnicas para reducir el estrés y un grupo control de lista de espera, para reducir la sintomatología física de los adolescentes. Los entrenamientos se aplicaron de forma colectiva y consistieron en 6 sesiones de intervención de dos horas cada una. Los participantes fueron 65 estudiantes de la ESO con edades entre los 13 y los 16 años. Los resultados muestran que el programa de entrenamiento centrado en el desarrollo de fortalezas personales es más eficaz para mejorar la sintomatología física que otro programa de entrenamiento y que la ausencia del mismo.
\end{abstract}

Palabras clave: adolescentes, bienestar, fortalezas personales, optimismo, síntomas físicos

\begin{abstract}
The intention is to compare the efficacy of an intervention program focused on training personal strengths, with respect to another intervention program based on training social skills and techniques to reduce stress and a control group on the waiting list, to reduce physical symptoms of adolescents. The trainings were applied collectively and consisted of six intervention sessions of two hours each. The participants were 65 secondary school students aged between 13 and 16 years. The results show that the training program focused on the development of personal strengths is more effective in improving physical symptoms than other training program or the absence of it.
\end{abstract}

Keywords: adolescents, wellness, personal strengths, optimism, physical health

\footnotetext{
${ }^{1}$ Psicólogo de la Salud, Máster en Psicología de la Salud y Doctorando en Psicología por la Universidad de Jaén, España. Tel.: 953757828. Correo: juanmolinapsicologoubeda@gmail.com

2 Profesora Titular de la Universidad de Jaén, España. Coordinadora del Máster de Psicología Positiva. Tel.: 953213368. Correo: angarcia@ujaen.es

Revista Iberoamericana de Diagnóstico y Evaluación - e Avaliação Psicológica. RIDEP · No60 $\cdot$ Vol.3 $\cdot 105-116 \cdot 2021$

ISSN: 1135-3848 print /2183-6051online
} 


\section{Introducción}

La adolescencia es una época de grandes cambios cognitivos, físicos, sociales y sexuales que puede propiciar la aparición y/o el aumento de síntomas físicos, debido a una mayor vulnerabilidad y a una mala adaptación de los adolescentes a estos profundos cambios (MarttilaTornio et al., 2020). En ésta etapa pueden aumentar problemas en la alimentación, como la bulimia y la anorexia nerviosa (Franko et al., 2005). Los datos recogidos en la encuesta de salud pública europea del año 2018 indican que el 5\% de los adolescentes europeos presentan problemas físicos. En el caso concreto de España, según la encuesta nacional de salud pública del año 2017, son alrededor de 215.000 los que sufren síntomas físicos (ENS, 2017).

Es bien sabido que la mayoría de las personas se enfrentarán a problemas de salud a lo largo de su vida y la evidencia científica muestra que los factores psicológicos son relevantes para la recuperación y la mejora de la salud (Broffman \& López 2011). Uno de estos factores es el optimismo disposicional, que constituye una creencia generalizada de que las cosas van a salir bien (Scheier \& Carver, 1985). Según diversas investigaciones, el optimismo disposicional mejora el bienestar físico, facilita el desarrollo de hábitos saludables, reduce los síntomas físicos de la enfermedad e incluso puede prolongar la vida (Carver \& Scheier, 2014).

Existe otra conceptualización sobre el optimismo la que lo considera como un estilo explicativo o atributivo (Peterson \& Seligman, 1984). El estilo explicativo es considerado como una tendencia (más o menos estable) referida al tipo de explicaciones que se realizan con respecto a las cosas que suceden en la vida diaria, tanto positivas como negativas. En la realización de estas atribuciones se utilizan tres dimensiones descriptivas: internalidad-externalidad, estabilidad -inestabilidad y globalidad-especificidad. Las personas con un estilo explicativo optimista suelen considerar los problemas o acontecimientos negativos como inestables, específicos y debidos a causas externas y los positivos suelen estar basados en atribuciones internas, estables y globales (Londoño et al., 2013). Éstas personas suelen aceptar mejor los reveses de la vida y se muestran más fuertes y resistentes a los efectos psicológicos y biológicos del estrés y las enfermedades (Sanjuán, 2007). Las investigaciones muestran que el estilo explicativo optimista está relacionado con una mejor salud física en adolescentes (Dingfelder, 2002).

Otro de los factores relevantes para la salud es el bienestar. Este se ha estudiado desde dos aproximaciones teóricas, la perspectiva hedónica o bienestar subjetivo y la perspectiva eudaimónica o bienestar psicológico. Son muchos los estudios que informan de una relación positiva entre el bienestar subjetivo y la salud física en distintas poblaciones (Brattberg, 1994; Holden et al., 1994; Langeveld et al., 1999). Con los adolescentes en concreto, se ha comprobado que aquéllos que sufren problemas de salud física, como migrañas, dolores de cabeza o estómago, presentan menos bienestar subjetivo que los que no presentan esos problemas de salud (Langevald et al., 1996; Moutinho et al., 2019). Por su parte, el bienestar psicológico ha ido ganando relevancia en los últimos años en las investigaciones realizadas con la salud física en distintas poblaciones (Steel et al., 2008). En cuanto a los adolescentes, se ha hallado también que este tipo de bienestar se relaciona de forma directa con la salud física, mostrando que los adolescentes que mantienen relaciones positivas con otras personas y se muestran autónomos y seguros de sí mismos informan de una mejor salud física percibida (Quiceno et al., 2014; Lira et al., 2019).

Durante las últimas décadas ha ido en aumento la investigación y aplicación de programas para mejorar la salud de los adolescentes. Estos programas ayudan a prevenir los problemas de salud y favorecen el aumento de la satisfacción y el bienestar de los adolescentes, así como un mejor ajuste al medio familiar, social y escolar (Hahn et al., 2007; Payton et al., 2008). Del mismo modo, distintas revisiones y metaanálisis (Durlak \& Wells, 1997; Greenberg et al., 2003; Wilson et al., 2003) han descrito importantes beneficios para los adolescentes como consecuencia de la participación en programas para mejorar la salud de los mismos.

Las investigaciones que muestran que el entrenamiento en fortalezas personales es eficaz para mejorar la salud psicológica son diversas tanto en población adulta como adolescente 
(Osborn et al., 2020; Wong et al., 2020). En el caso de la salud física se han encontrado investigaciones con adultos que se muestran eficaces (Mohammadi et al., 2017; Mollinari et al., 2018), siendo menores las encontradas en el caso de la población adolescente (Sherif et al., 2018).

Teniendo en cuenta las pocas investigaciones realizadas con adolescentes, este trabajo pretende comprobar la eficacia de un programa de intervención, centrado en fomentar fortalezas personales, sobre la salud física de los adolescentes. Además, se comprobará la eficacia diferencial de esta intervención con un grupo control y con otro grupo de entrenamiento en habilidades sociales y técnicas para reducir el estrés. Por último, se estudiará la eficacia de las dos intervenciones para mantener los efectos pasados 6 meses desde la intervención.

\section{Método}

\section{Diseño}

Para la realización de este estudio se parte de un diseño factorial mixto, con una variable entregrupos y una variable intrasujeto. La variable entregrupos ha sido el Grupo de Entrenamiento y tiene tres niveles: grupo de entrenamiento en fortalezas personales, grupo de entrenamiento en habilidades sociales y estrategias de afrontamiento del estrés y grupo control de lista de espera. La variable intrasujeto ha sido la Fase de Entrenamiento y ha constado igualmente de tres niveles para la mayoría de las variables dependientes: pretest (antes de comenzar el entrenamiento), postest (después de concluir las 6 sesiones de entrenamiento) y seguimiento (a los seis meses desde la conclusión del tratamiento).

\section{Participantes}

De un total de 300 adolescentes se seleccionaron 65 (33 chicos y 32 chicas), con un rango de edad que oscilaba entre 13 y 17 años de edad $(\mathrm{M}=14.32$ y $\mathrm{DT}=.89)$. Los participantes fueron estudiantes de $2^{\circ}$ y $3^{\circ}$ de E.S.O. de tres localidades de la provincia de Jaén. Fueron seleccionados aquellos adolescentes que obtuvieron un centil de 25 o menos en el estilo explicativo optimista-pesimista (CASQ) y en la puntuación de felicidad (OHS), tomando como base para el cálculo de los centiles los 300 adolescentes. Los participantes elegidos fueron distribuidos al azar a uno de los tres grupos del estudio. En el grupo 1 (control de lista de espera) fueron asignados 11 chicos y 11 chicas $(\mathrm{M}=14.27$ y $\mathrm{DT}=.83$ ). Al grupo 2 (entrenamiento en habilidades sociales y reducción del estrés) fueron asignados 11 chicos y 10 chicas $(M=14.24$ y $\mathrm{DT}=.70$ ). El grupo 3 (entrenamiento en fortalezas personales) quedó compuesto por 11 chicos y 11 chicas $(\mathrm{M}=14.45$ y $\mathrm{DT}=1.10)$. Los tres grupos eran homogéneos en cuanto a las variables sociodemográficas de edad y sexo, así como con respecto a posibles variables moderadoras de los resultados como hábitos de estudio, extraversión, estabilidad emocional e inteligencia; por último, también mostraban valores semejantes en los niveles de estilo explicativo y felicidad. Todos los adolescentes participaron voluntariamente en el estudio y se contó con su propio consentimiento y el de sus padres, así como con el de los consejos escolares y el de los equipos directivos de los centros (Véanse la Figura 1 y la Tabla 1).

\section{Instrumentos}

1. Cuestionario Revisado de Estilo Atribucional para Niños (CASQ) (Seligman et al., 1984), en su versión adaptada y traducida al castellano en la obra de Seligman (2011). Consta de 48 ítems y 6 subescalas: tres de ellas para los acontecimientos negativos en sus dimensiones temporal-permanente, global-específica e internaexterna, respectivamente, y otras tres para los eventos positivos y las mismas dimensiones. Cada respuesta interna, estable o global se puntúa con un uno, mientras que las respuestas externas, inestables o específicas se puntúan con un cero. Al sumar los valores obtenidos para las tres dimensiones positivas se obtiene una puntuación compuesta positiva $\mathrm{CP}$ y una negativa para las dimensiones negativas $\mathrm{CN}$. La puntuación total se obtiene de restarle al $\mathrm{CP}$ las puntuaciones del $\mathrm{CN}$. Los coeficientes de consistencia interna para las distintas subescalas son moderados, oscilando entre .44 y .78 (Seligman et al., 1984). La fiabilidad test-retest, mejora a medida que aumenta la edad, con un rango de puntuaciones que se sitúa entre .60 y .80 (Nolen-Hoeksema, 1992). Respecto a algunos datos de validación con población española, la consistencia interna para una estructura bifactorial (positivos-negativos) 
Tabla 1. Medias, desviaciones típicas y ANOVAS de las variables edad, sexo, hábitos de estudio, felicidad, estilo explicativo, extraversión, estabilidad emocional e inteligencia en función de los grupos

\begin{tabular}{|c|c|c|c|c|c|c|c|c|c|}
\hline \multirow[b]{2}{*}{ Variable } & \multicolumn{3}{|c|}{ Media } & \multicolumn{3}{|c|}{ Desviación Típica } & \multirow[b]{2}{*}{$\mathrm{F}$} & \multirow[b]{2}{*}{$p$} & \multirow[b]{2}{*}{$\eta^{2} p$} \\
\hline & GC1 & GHE2 & GFP3 & GC1 & GHE2 & GFP3 & & & \\
\hline Sexo & & & & & & & .015 & .694 & .012 \\
\hline Edad & 14.27 & 14.24 & 14.45 & .83 & .70 & 1.10 & .367 & .985 & .000 \\
\hline H.E escala 1 & 18.82 & 18.45 & 19.55 & 3.72 & 4.97 & 3.84 & .379 & .686 & .012 \\
\hline H.E escala 2 & 8.91 & 8.31 & 9.52 & 4.50 & 4.87 & 3.48 & .425 & .656 & .014 \\
\hline H.E escala 3 & 14.45 & 12,83 & 14.86 & 2.94 & 2.97 & 2.60 & 2.783 & .070 & .082 \\
\hline H.E escala 4 & 19.07 & 18.07 & 16.93 & 3.08 & 2.90 & 3.48 & 2.508 & .090 & .075 \\
\hline Extraversión & 21 & 22.62 & 23.59 & 6 & 8.43 & 8.59 & .626 & .538 & .020 \\
\hline E. E & 39.32 & 35.86 & 32.68 & 6.83 & 9.65 & 10.05 & 3.028 & .056 & .089 \\
\hline Inteligencia & 22.95 & 20.14 & 22.18 & 5.35 & 7.83 & 4.84 & 1.206 & .306 & .037 \\
\hline Felicidad & 59.55 & 66.62 & 57.68 & 16.98 & 12.94 & 12.43 & 2.327 & .106 & .070 \\
\hline E.E.P & 14.05 & 12.95 & 14.05 & 4.36 & 3.37 & 4.03 & .478 & .623 & .015 \\
\hline E.E.N & 15.27 & 14.14 & 14.95 & 2.51 & 1.15 & 2.36 & 1.272 & .288 & .039 \\
\hline E.E Total & -.86 & -1.19 & -.91 & 4.61 & 3.76 & 3.56 & 2.068 & .135 & .063 \\
\hline
\end{tabular}

*GC1: Grupo Control 1, GHE2: Grupo de estrés y habilidades sociales 2, GFP3: Grupo de Fortalezas Personales 3, H.E Escala 1: Hábitos de Estudio Escala de Condiciones ambientales, H.E Escala 2 Hábitos de Estudio Escala de Planificación del estudio, H.E Escala 3, Hábitos de Estudio Escala de Utilización de materiales, H.E Escala 4 Escala de Hábitos de Estudio Escala de Asimilación de contenidos; E. E: Estabilidad Emocional, .E. E.P Estilo Explicativo para los pensamientos positivos, E.E.N Estilo Explicativo para los pensamientos negativos, E.E Total Estilo Explicativo para el total.

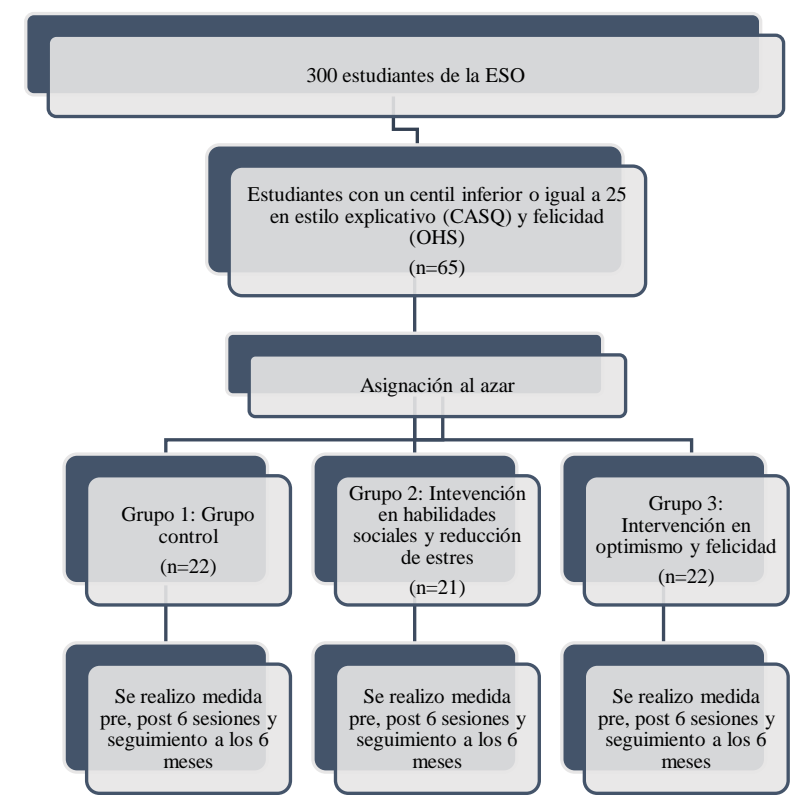

Figura 1. Diagrama de flujo de los participantes del estudio

indica valores de .85 y .90 respectivamente. Por último, los valores del alfa de Cronbach obtenidos con la muestra del estudio para las seis subescalas oscilan entre .59 y .62 .

2. Escala de Felicidad de Oxford (OHS) (Hills $\&$ Argyle, 2002). Mide los aspectos motivacionalconductuales, afectivo-cognitivos y fisiológicos de la felicidad individual o bienestar subjetivo, a través de 29 elementos que se puntúan en una escala de 1 a 6 . El alfa de Cronbach hallado para esta escala ha sido de $.84 \mathrm{y}$ el valor de fiabilidad test-retest de .78 (Peterson et al., 2005). En cuanto a la validez convergente y discriminante, la puntuación del OHS muestra una correlación positiva de .32 con la Escala de Afectividad Positiva de Bradburn y de .57 con la escala de Satisfacción Vital de Diener (SWLS). Por último, el valor de consistencia interna calculado con esta muestra es de.95.

3. Escala de Síntomas Somáticos Revisada (ESS-R) (Sandin \& Chorot, 1991). La ESS-R es una escala de 54 ítems desarrollada para evaluar diversas quejas somáticas y problemas de salud. Los sujetos deben contestar con un rango de respuesta de 1 ("Nunca") a 5 ("Más de una vez al mes"). La ESS-R contiene 9 subescalas que hacen referencia a los siguientes sistemas físicos: inmunológico general, cardiovascular, respiratorio, gastrointestinal, neurosensorial, piel y alergia, musculoesquelético, genitourinario y reproductor femenino. El rango de respuesta para la consistencia interna de las distintas subescalas varía entre .81 y .84 . En cuanto a los datos sobre consistencia temporal (un mes), las escalas presentan puntuaciones situadas entre .73 y .82 (Sandín \& Chorot 1991). Las puntuaciones del alfa de Cronbach en este estudio oscilan entre .77 y.84.

4. Cuestionario de Personalidad Big Five para niños y adolescentes (BFQ-NA) (Barbaranelli et al., 1998), en la versión española de TEA, traducida al castellano por Del Barrio, Carrasco y Holgado (2006). El objetivo del instrumento es evaluar cinco dimensiones de la personalidad: responsabilidad, amabilidad, estabilidad emocional, extraversión y apertura. 
Tabla 2. Sesiones, objetivos y actividades del entrenamiento en fortalezas

\begin{tabular}{ll}
\hline Sesiones & \multicolumn{1}{c}{ Objetivos } \\
\hline $\begin{array}{l}\text { Sesión 1: Terapia } \\
\text { cognitiva y }\end{array}$ & $\begin{array}{l}\text { Conocer la terapia cognitiva y el pensamiento } \\
\text { optimista }\end{array}$ \\
$\begin{array}{l}\text { Optimismo } \\
\text { Sesión 2: Pesimismo }\end{array}$ & $\begin{array}{l}\text { Conocer las implicaciones y consecuencias que } \\
\text { tiene el pensamiento pesimista }\end{array}$
\end{tabular}

Sesión 3: Cambiar el pensamiento

Sesión 4: Ser uno mismo
Saber sustituir un pensamiento pesimista por uno optimista

Conocer la importancia de ser uno mismo y de apreciar la felicidad

Saber organizarse mejor y no agobiarse objetivos y gestionar el tiempo
Sesión 6: Ser más activo
Aumentar la actividad y la vida social
Introducción a la terapia cognitiva

Modelo ABC de Ellis (A son los acontecimientos, B las creencias de la personas y $\mathrm{C}$ las consecuencias) ¿Qué es el pensamiento optimista?

Ejemplos de pensamientos optimistas y sus consecuencias

Autorregistro de acontecimientos, pensamientos positivos y sus consecuencias

Corrección del autorregistro anterior

¿Qué es el pensamiento pesimista?

Ejemplos de pensamientos pesimistas y sus consecuencias

Autorregistro de acontecimientos, pensamientos negativos y sus consecuencias

Corrección del autorregistro anterior

¿Se puede sustituir el pensamiento?

Generar alternativas optimistas a los pensamientos pesimistas

Poner en práctica la sustitución en el entorno escolar y familiar

Explicar la importancia de ser uno mismo y de apreciarse tal y como se es

Pensar cosas buenas sobre uno mismo

Escribir cartas de gratitud y de perdón

Practicar la responsabilidad

Hacer más actividades sociales

Practicar en el colegio y en el entorno familiar

Corregir la práctica de la sesión anterior

Diseñar objetivos y las acciones necesarias para conseguirlos

Utilizar listas y agendas

Conocer la procrastinación y tomar conciencia de ella para evitarla

Practicar en el entorno

Corregir la práctica anterior

Ver qué actividades producen más felicidad (tanto individuales como sociales)

Diseñar acciones para aumentar las actividades sociales
Consta de 65 ítems que se valoran mediante una escala de cinco alternativas. En la versión española el instrumento presenta buenas propiedades psicométricas, encontrándose un coeficiente alfa que oscila entre .78 y .86 para las distintas subescalas (Carrasco \& Del Barrio, 2006). En nuestro caso únicamente se tendrán en cuenta las subescalas de extraversión (alfa de Cronbach de .82) y estabilidad emocional (alfa de Cronbach de .84). En este estudio se obtuvo un alfa de Cronbach de .76 para la subescala de extraversión y .82 para la de estabilidad emocional.

5. Inventario de Hábitos de Estudio (IHE) de Pózar. Es un instrumento que pretende evaluar los hábitos de trabajo y de estudio mediante cuatro escalas: condiciones ambientales del estudio, planificación del estudio, utilización de materiales y asimilación de contenidos. Consta de 90 ítems con tres alternativas de respuesta. Según el estudio de validación con muestras españolas, la prueba muestra buenos índices de fiabilidad dos mitades (valores en torno a .90) y de validez de criterio (valores en torno a .80). Las puntuaciones del alfa de Cronbach en este estudio oscilan entre $.84 \mathrm{y}$ 88 .

6. D-70. Es una prueba que mide la inteligencia general evaluando las funciones de abstracción y comprensión de relaciones. Se ha utilizado la adaptación española llevada a cabo por Cruz, Cordero, Seisdedos y González del año 1971. Esta prueba consta de 44 ítems, en los que se presentan varias fichas de dominós ordenadas según una secuencia lógica que el sujeto debe 
Tabla 3. Sesiones, objetivos y actividades del entrenamiento en habilidades sociales y técnicas de reducción del estrés

\begin{tabular}{|c|c|c|}
\hline Sesiones & Objetivos & Actividades \\
\hline $\begin{array}{l}\text { Sesión 1: Habilidades } \\
\text { interpersonales }\end{array}$ & $\begin{array}{l}\text { Conocer las } \\
\text { habilidades } \\
\text { interpersonales y } \\
\text { sus ventajas }\end{array}$ & $\begin{array}{l}\text { Explicar qué son las habilidades interpersonales, las ventajas de tenerlas } \\
\text { y las desventajas de carecer de ellas } \\
\text { La comunicación verbal y no verbal } \\
\text { Comunica sin hablar } \\
\text { Puesta en práctica en situaciones sociales }\end{array}$ \\
\hline $\begin{array}{l}\text { Sesión 2: Estilos de } \\
\text { comunicación }\end{array}$ & $\begin{array}{l}\text { Conocer los tres } \\
\text { estilos } \\
\text { comunicativos y } \\
\text { sus diferencias }\end{array}$ & $\begin{array}{l}\text { Corrección de la puesta en práctica de la sesión anterior } \\
\text { Los derechos de los asertivos } \\
\text { Aprender a decir no } \\
\text { Puesta en práctica del estilo asertivo }\end{array}$ \\
\hline $\begin{array}{l}\text { Sesión 3: Conectar con los } \\
\text { demás }\end{array}$ & $\begin{array}{l}\text { Saber pedir una } \\
\text { cita y hacer } \\
\text { cumplidos }\end{array}$ & $\begin{array}{l}\text { Corrección de la práctica del estilo asertivo } \\
\text { Iniciar, mantener y finalizar una conversación } \\
\text { Dar y recibir elogios y cumplidos } \\
\text { Poner en práctica la sustitución en el entorno escolar y familiar }\end{array}$ \\
\hline $\begin{array}{l}\text { Sesión 4: Respiración } \\
\text { diafragmática }\end{array}$ & $\begin{array}{l}\text { Saber utilizar la } \\
\text { respiración } \\
\text { diafragmática }\end{array}$ & $\begin{array}{l}\text { Corrección de la puesta en marcha de la sesión anterior } \\
\text { Breve explicación de la respiración diafragmática y de sus características } \\
\text { e implicaciones } \\
\text { Inspiración/expiración con el diafragma } \\
\text { Práctica diaria }\end{array}$ \\
\hline Sesión 5: Relajación & $\begin{array}{l}\text { Saber utilizar la } \\
\text { relajación como } \\
\text { forma de } \\
\text { desactivarse }\end{array}$ & $\begin{array}{l}\text { Corregir la práctica de la sesión anterior } \\
\text { Relajación de Jacobson } \\
\text { Relajación pasiva } \\
\text { Práctica diaria }\end{array}$ \\
\hline Sesión 6: Autoinstrucciones & $\begin{array}{l}\text { Saber utilizar las } \\
\text { autoinstrucciones }\end{array}$ & $\begin{array}{l}\text { Corregir la práctica de la sesión anterior } \\
\text { Explicación de qué son las autoinstrucciones } \\
\text { Autoinstrucciones de Meichenbaum } \\
\text { Práctica diaria }\end{array}$ \\
\hline
\end{tabular}

descubrir y escribir en el lugar adecuado. En cuanto a las propiedades psicométricas, la prueba muestra una fiabilidad dos mitades de .82, así como una correlación de. 47 con la puntuación que se obtiene en el Test de Aptitudes PMA y de .62 en relación con el Test de inteligencia no verbal de Bonnarel (B-53). Las puntuaciones del alfa de Cronbach en este estudio oscilan entre .78 y.82.

\section{Procedimiento}

El estudio constó de tres fases que se aplicaron a los tres grupos: 1) Evaluación pretratamiento, en la cual se administraron las siguientes pruebas CASQ, OHS, BFQ-NA, ESS$\mathrm{R}$, IHE, D70 (todas las pruebas fueron contrabalanceadas en cada una de las fases y se aplicaron de forma colectiva en el aula asignada y en el horario de tutorías de cada grupo). 2) Aplicación de los programas de intervención (los participantes se dividieron en tres subgrupos para aplicarles el entrenamiento correspondiente. (Véanse las Tablas 2 y 3 con las sesiones de entrenamiento concretas y el contenido de las mismas). Tras la conclusión de las intervenciones, se aplicaron a todos los grupos los siguientes instrumentos IHE, CASQ, OHS, ESS-R. 3) Seguimiento a los seis meses desde la finalización de las intervenciones, en la que se aplicaron nuevamente a los tres grupos los siguientes instrumentos CASQ, OHS Y ESS-R.

\section{Análisis de datos}

Para determinar si existían diferencias entre los distintos grupos en la fase pretratamiento, se llevó a cabo un ANOVA de un factor (Grupo) con las variables edad, sexo, hábitos de estudio (distintas escalas), felicidad, estilo explicativo (para los acontecimientos positivos y negativos) extraversión, estabilidad emocional e inteligencia. Por su parte, para comprobar las diferencias de Grupo en las variables dependientes se realizó un Análisis de Covarianza (ANCOVA), tomando como variable independiente la variable Grupo, con tres niveles 


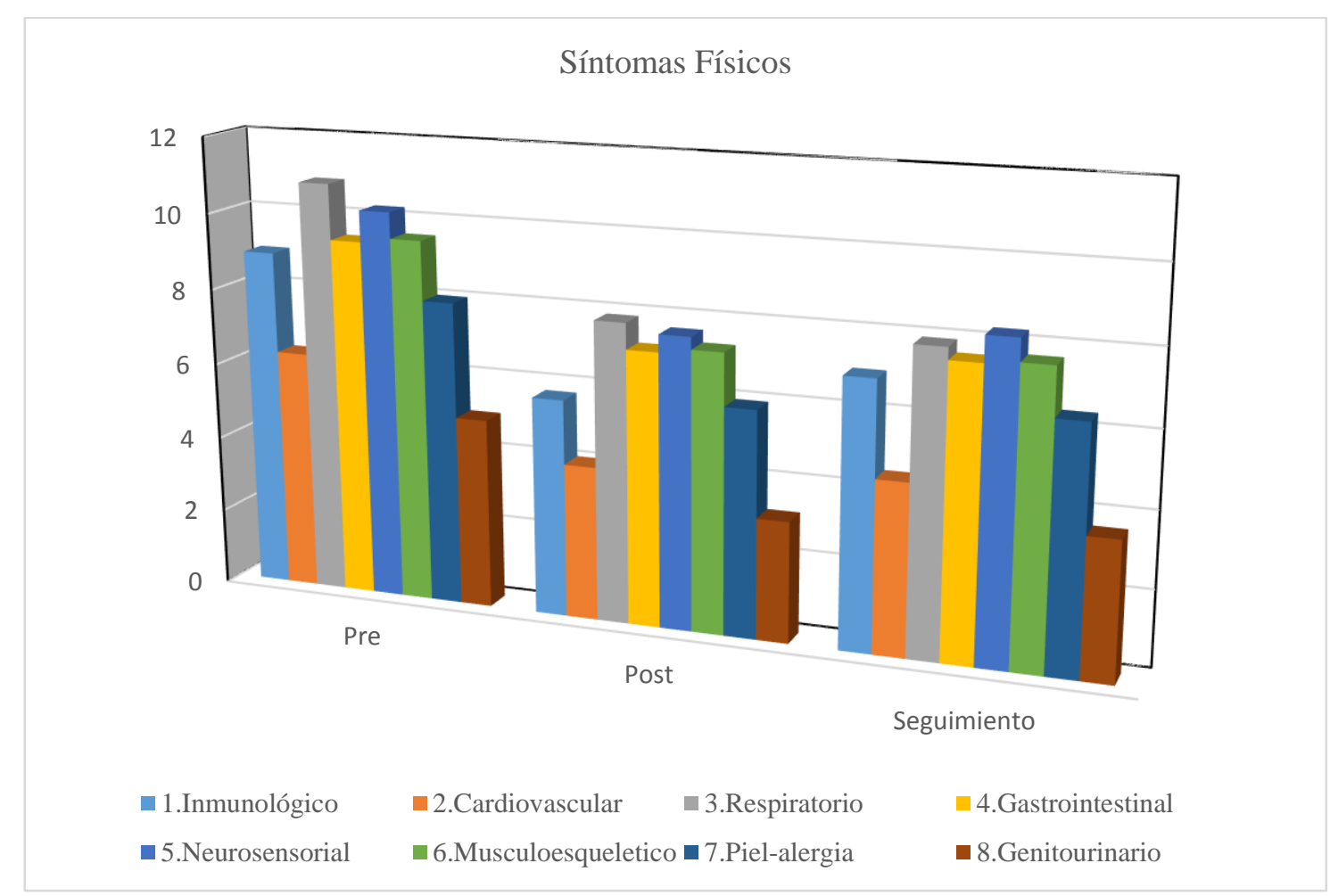

Figura 2. Efectos del entrenamiento sobre las subescalas de los síntomas físicos durante las distintas fases de entrenamiento

(entrenamiento en fortalezas, entrenamiento en habilidades sociales y reducción del estrés y control de lista de espera), y como covariante la fase pre de cada grupo. No se tuvieron en cuenta las puntuaciones directas de las variables dependientes, sino que se tomó como variables de medida post las puntuaciones diferenciales resultantes de restar la fase pre de cada grupo a su propia fase post, y como variables de medida de seguimiento las puntuaciones diferenciales resultantes de restar la fase post de cada grupo a su propia fase de seguimiento. Para todos los contrastes a posteriori entre los grupos se utilizó la prueba de Bonferroni. El tamaño del efecto se calculó utilizando la delta de Cohen a partir de la fórmula de la diferencia media tipificada.

\section{Resultados}

\section{Resultados en la fase pretratamiento}

Los resultados del ANOVA no mostraron, en ninguna de las variables de estudio, diferencias significativas entre los grupos en la fase pretratamiento.

\section{Resultados tras el entrenamiento $y$ en el seguimiento}

Los efectos conseguidos tras el entrenamiento en fortalezas, en las diferentes subescalas de síntomas físicos, en comparación con el grupo control y con el grupo de estrés y habilidades sociales son los siguientes (Véanse la Figura 2 y la Tabla 4).

En lo que respecta a los síntomas del sistema inmunológico se obtiene un efecto significativo para la variable grupo tras las 6 sesiones de tratamiento. Las comparaciones por parejas realizadas en los grupos muestran diferencias significativas entre los grupos de control y de fortalezas (3) ( $p<.001)$, con tamaño del efecto alto $\left(d_{2}=-1.31\right)$ y entre los grupos de estrés y habilidades sociales (2) y de fortalezas (3) $(p<.001)$, con un tamaño de efecto igualmente alto $\left(d_{3}=-1.00\right)$, siendo menores los síntomas en el grupo de fortalezas que en los otros dos grupos. Los resultados relativos al seguimiento no mostraron efectos significativos de Grupo.

En referencia al sistema cardiovascular se encuentran efectos significativos para la variable grupo. Las comparaciones por parejas realizadas en los grupos muestran diferencias 
Tabla 4. Medias, desviaciones típicas y datos estadísticos de los síntomas físicos en función de los grupos

\begin{tabular}{|c|c|c|c|c|c|c|c|c|c|c|}
\hline \multirow[t]{2}{*}{$\mathrm{VB}$} & \multirow[t]{2}{*}{$\mathrm{FE}$} & \multirow{2}{*}{$\begin{array}{c}\text { G3-FP } \\
\text { M (DT) }\end{array}$} & \multirow{2}{*}{$\begin{array}{l}\text { G2-HE } \\
\text { M (DT) }\end{array}$} & \multirow{2}{*}{$\begin{array}{c}\text { G1-C } \\
\mathrm{M}(\mathrm{DT})\end{array}$} & \multicolumn{6}{|c|}{ Datos Estadisticos Globales } \\
\hline & & & & & $\mathrm{F}$ & $p$ & $\eta^{2} p$ & $d_{3}$ & $d_{2}$ & $d_{1}$ \\
\hline \multirow[t]{2}{*}{ IN } & Pre-6 & $-3.22(2.83)$ & $-.39(1.55)$ & $-.32(1.21)$ & 22.30 & $.000 * *$ & .422 & & & \\
\hline & 6/S & $1.27(1.88)$ & $.48(1.03)$ & $.50(1.37)$ & 1.61 & .207 & .050 & -1.00 & -1.31 & -.42 \\
\hline \multirow[t]{2}{*}{$\mathrm{CV}$} & Pre-6 & $-2.26(2.19)$ & $.10(6.30)$ & $.18(.50)$ & 23.90 & $.000 * *$ & .439 & & & \\
\hline & $6 / \mathrm{S}$ & $.45(1.26)$ & $.29(.72)$ & $-.13(.64)$ & 3.32 & $.043 *$ & .098 & -.22 & -.70 & -.27 \\
\hline \multirow[t]{2}{*}{$\mathrm{RP}$} & Pre-6 & $-3.00(4.27)$ & $-.09(5.71)$ & $-.37(1.33)$ & 11.80 & $.000 * *$ & .279 & & & \\
\hline & $6 / \mathrm{S}$ & $.05(1.33)$ & $.28(.78)$ & $.73(2.05)$ & 2.52 & .089 & .076 & -.04 & -.83 & -.67 \\
\hline \multirow[t]{2}{*}{ GI } & Pre-6 & $-2.23(1.85)$ & $-.58(3.27)$ & $-.05(.72)$ & 22.76 & $.000 * *$ & .427 & & & \\
\hline & $6 / S$ & $.41(1.01)$ & $.48(1.12)$ & $.19(1.18)$ & .41 & .667 & .013 & -.05 & -1.53 & -.85 \\
\hline \multirow[t]{2}{*}{ NS } & Pre-6 & $-2.59(2.17)$ & $-.33(.32)$ & $-.22(1.38)$ & 22.57 & $.000^{* * *}$ & .403 & & & \\
\hline & $6 / S$ & $.63(1.81)$ & $.33(1.02)$ & $-.28(.94)$ & 3.10 & .052 & .092 & -1.61 & -1.27 & -.22 \\
\hline \multirow[t]{2}{*}{ ME } & Pre-6 & $-2.23(1.74)$ & $-.24(.48)$ & .77 (1.69) & 26.56 & $.000^{* * *}$ & .465 & & & \\
\hline & 6/S & $.36(.79)$ & $.24(.62)$ & $.01(.87)$ & .843 & .435 & .027 & -1.60 & -.83 & -.50 \\
\hline \multirow[t]{2}{*}{ PA } & Pre-6 & $-2.05(1.84)$ & $-.10(.36)$ & $.45(.74)$ & 30.55 & $.000 * *$ & .500 & & & \\
\hline & $6 / \mathrm{S}$ & $.46(1.22)$ & $.33(.58)$ & $-.04(.95)$ & 1.36 & .263 & .043 & -1.41 & -1.12 & -.52 \\
\hline \multirow[t]{2}{*}{ GU } & Pre-6 & $-1.82(1.74)$ & $-.05(.47)$ & $.23(.53)$ & 30.18 & $.000 * *$ & .497 & & & \\
\hline & $6 / \mathrm{S}$ & $.46(.96)$ & $.05(.67)$ & $.18(.50)$ & 1.823 & .170 & .056 & -1.31 & -1.21 & .02 \\
\hline
\end{tabular}

$* p<.05 ; * * p<.01 ; \mathrm{VB}=$ Variables, $\mathrm{FE}=$ Fase de entrenamiento, G3-FP= Grupo fortalezas personales, G2-HE=Grupo de estrés y habilidades sociales, G1-C=Grupo control, d3=grupo optimismo-grupo habilidades sociales; d2=grupo optimismo - grupo control; d1=grupo de habilidades sociales- grupo control. IN=inmunológico; $\mathrm{CV}=$ cardiovascular; $\mathrm{RP}=$ respiratorio; $\mathrm{GI}=$ gastrointestinal; NS= neurosensorial; $\mathrm{ME}=$ musculo-esquelético; PA=piel-alergia; $\mathrm{GU}=$ genitourinario. Pre-6=puntuaciones diferenciales entre la fase pre y la fase post 6 sesiones; $6 / \mathrm{S}=$ puntuaciones diferenciales entre la fase post 6 sesiones y el seguimiento

significativas entre los grupos control (1) y fortalezas (3) $(p<.001)$, con un tamaño de efecto alto $\left(d_{2}=-.70\right)$ y entre los grupos estrés y habilidades sociales (2) y fortalezas (3) $(p<.001)$, con un tamaño de efecto bajo $\left(d_{3}=-.22\right)$, siendo los síntomas más bajos en el grupo que recibió el entrenamiento en fortalezas. Además, los resultados de este análisis en el período de seguimiento mostraron igualmente efectos significativos para la variable de grupo. Las comparaciones por parejas realizadas en los grupos indican diferencias significativas entre los grupos control (1) y fortalezas (3) $(p<.001)$ y entre los grupos estrés y habilidades sociales (2) y fortalezas (3) $(p<.001)$. Aunque las puntuaciones se incrementaban con respecto a la fase de tratamiento, los síntomas todavía seguían siendo más bajos en el grupo de fortalezas que en los otros dos grupos del estudio.

Los resultados del sistema respiratorio muestran diferencias significativas para la variable grupo. Las comparaciones por parejas realizadas en los grupos señalan diferencias significativas entre los grupos control (1) y fortalezas (3) $(p<.001)$, con tamaño de efecto alto $\left(d_{2}=-.83\right)$ y entre los grupos estrés y habilidades sociales (2) y fortalezas (3) $(p<.001)$, con tamaño del efecto nulo $\left(d_{3}=-.04\right)$. Los síntomas fueron menores en el grupo experimental de fortalezas. Posteriormente, los resultados de los análisis realizados sobre la fase de seguimiento no mostraron efectos significativos para la variable de grupo.

Los resultados para el sistema gastrointestinal muestran efectos significativos para la variable de grupo. Las comparaciones por parejas realizadas en los grupos exhiben diferencias significativas entre los grupos control (1) y fortalezas (3) $(p<.001)$, con tamaño de efecto alto $\left(d_{2}=-.1 .53\right), \mathrm{y}$ entre los grupos estrés y habilidades sociales (2) y fortalezas (3) $(p<.001)$, con tamaño del efecto nulo $\left(d_{3}=-.05\right)$. Es el grupo que ha recibido el entrenamiento en fortalezas el que muestra menores puntuaciones en sintomatología gastrointestinal que los otros dos. Además, los resultados del análisis durante la fase de seguimiento no mostraron efectos significativos para la variable de grupo.

En referencia al sistema neurosensorial los resultados muestran efectos significativos para la variable grupo. Las comparaciones por parejas realizadas muestran diferencias significativas entre los grupos control (1) y fortalezas (3) $(p<.001)$, con tamaño del efecto alto $\left(d_{2}=-1.27\right)$, y entre los grupos estrés y habilidades sociales (2) y fortalezas (3) $(p<.001)$, igualmente con tamaño 
del efecto alto $\left(d_{3}=-1.61\right)$, recogiéndose las puntuaciones más bajas en los síntomas en el grupo de fortalezas. Finalmente, los resultados durante el seguimiento no mostraron efectos significativos para la variable de grupo.

Los resultados para el sistema musculo esquelético muestran efectos significativos para la variable de grupo. Las comparaciones por parejas realizadas en los grupos exhiben diferencias significativas entre los grupos control (1) y fortalezas (3) $(p<.001)$, siendo el tamaño del efecto alto $\left(d_{2}=-.83\right)$. En concreto, el grupo que ha recibido el entrenamiento en fortalezas muestra menor nivel de sintomatología que el grupo de control de lista de espera. Posteriormente, los resultados del análisis estadístico en el período de seguimiento no mostraron efectos significativos para la variable grupo.

Los resultados sobre la variable piel alergia después de las 6 sesiones de entrenamiento indicaron efectos significativos para la variable de grupo. Las comparaciones por parejas realizadas en los grupos muestran diferencias significativas entre los grupos control (1) y fortalezas (3) $(p<.001)$, con tamaño del efecto alto $\left(d_{2}=-1.12\right)$, y entre los grupos estrés y habilidades sociales (2) y fortalezas (3) $(p<.001)$ con tamaño del efecto también alto $\left(d_{3}=-1.41\right)$, siendo los síntomas del sistema más bajos en el grupo de fortalezas. Al igual que en casos anteriores, los resultados durante el seguimiento no mostraron efectos significativos para la variable de grupo.

Para finalizar, s los efectos encontrados para el sistema genitourinario revelan igualmente efectos significativos para la variable de grupo. Las comparaciones por parejas realizadas en los grupos muestran diferencias significativas entre los grupos control (1) y fortalezas (3) $(p<.001)$, con tamaño del efecto alto $\left(d_{2}=-1.21\right)$, y entre los grupos estrés y habilidades sociales (2) y fortalezas (3) $(p<.001)$, con tamaño del efecto igualmente alto $\left(d_{3}=-1.31\right)$. El nivel de sintomatología fue menor en el grupo de fortalezas. Finalmente, los resultados no mostraron efectos significativos para la variable de grupo en el período de seguimiento.

\section{Discusión}

Tras la aplicación del tratamiento, los resultados muestran que los participantes del grupo que recibió el entrenamiento en fortalezas presentan menores niveles de sintomatología física que los otros dos grupos en todas las subescalas evaluadas; por su parte, no se encuentran diferencias en ninguna de las subescalas de síntomas físicos entre los grupos de habilidades sociales y control de lista de espera. En cuanto al seguimiento, los resultados muestran que los participantes entrenados en fortalezas personales mantienen los efectos conseguidos tras la intervención en las subescalas de sintomatología cardiovascular, respiratoria, gastrointestinal, neurosensorial y piel-alergia.

Los datos mencionados son similares a los hallados en otros trabajos publicados, en los cuales se muestra que el entrenamiento en fortalezas similares a las que se han entrenado en nuestro caso es más eficaz que otro tipo de entrenamiento, o la ausencia del mismo, para reducir los síntomas físicos tanto en población adulta (Mohammadi et al., 2017; Mollinari et al., 2018) como en adolescentes (Briones, 2018); y para mantener estos resultados a lo largo del tiempo (Remor \& Amorós, 2012).

Estos resultados son prometedores de cara a establecer programas de intervención que ayuden a minimizar y/o prevenir los problemas de salud física de los adolescentes. El entrenamiento en fortalezas se muestra más eficaz que el entrenamiento en habilidades sociales y técnicas para reducir el estrés, por lo que centrarse en este entrenamiento puede ayudar a realizar intervenciones más efectivas, más cortas y menos costosas. El hecho de no mostrarse eficaz el entrenamiento en estrés y habilidades sociales muestra la poca relevancia de esta intervención de cara a mejorar y/o prevenir la salud física de los adolescentes.

Incluir éstos programas en el diseño de los planes educativos tendría sentido ya que podrían aplicarse a lo largo de todo el curso escolar o en determinados momentos. Implantarlos de forma transversal, en los centros educativos, podría mejorar la educación en salud, ayudar a un desarrollo más completo y amplio del adolescente, mejorar y/o prevenir su salud física. Para ello se 
podrían entrenar al profesorado para que fuesen ellos los que impartieran los entrenamientos. Una vez que los profesores estuviesen formados para poder impartir los tratamientos con garantías, éstos se podrían llevar a cabo, de forma semanal, en los horarios de tutoría y a lo largo de todo el curso académico.

A pesar de los resultados obtenidos, el estudio ofrece resultados preliminares y presenta algunas limitaciones como las siguientes:

En primer lugar el tamaño de la muestra. Veintidós alumnos por grupo es un número escaso que puede plantear dificultades en la representatividad y la generalización de los resultados. En estudios posteriores podría realizarse un muestreo más representativo que abarcase centros de las distintas zonas geográficas, además de ampliar el número de participantes.

En segundo lugar, el instrumento de evaluación utilizado para medir los síntomas físicos. Se encontraron dificultades de comprensión en algunos de los ítems. Para resolver este problema en estudios posteriores podría darse una explicación previa sobre las dificultades de comprensión concretas que ya se sabe que podrían producirse antes de que los participantes rellenen el instrumento. Igualmente, puede insistirse a los participantes en la importancia de preguntar todo aquello que no se entienda, evitando que contesten sin saber lo que se les está preguntando. Por último, puesto que en la evaluación de los síntomas físicos el instrumento de medida es de autoinforme, podría complementarse la información mediante informes de terceras personas como padres y profesionales clínicos.

En tercer lugar el momento del periodo de seguimiento. Este periodo se realizó en el mes de septiembre, mes que se caracteriza porque el alumnado se incorpora al nuevo curso académico después de un periodo de dos meses de descanso, en el cual han tenido tiempo de reducir el estrés y la ansiedad que le pueda provocar la presión del curso académico anterior, así como descansar físicamente; además de ser un mes donde se renuevan las ganas y la ilusión con la entrada del nuevo curso. Estos elementos pueden influir temporalmente en la percepción que tienen los alumnos sobre su sintomatología física y aparecer reflejado en la evaluación realizada. Por lo tanto, quizá sería mejor llevar a cabo el seguimiento dentro del mismo curso académico y/o ampliar su duración para que coincida con el periodo escolar del siguiente curso.

Para finalizar se presenta una dirección para futuras investigaciones con respecto a la recogida de datos y es la dificultad de acceso a la muestra para la aplicación del entrenamiento. Sería conveniente realizarlo en horario no escolar, debido a que los participantes suelen ser de distintas clases y es realmente complicado poder reunirlos para realizar las sesiones de intervención durante el horario escolar.

\section{Referencias}

Barbaranelli, C., Caprara, G. V., \& Rabasca, A. (1998). Manuale del BFQC. Big Five Questionnaire Children. O.S. Organizzaaioni Speciali-Firenze. Grupo de pertenencia pronosticado.

Brattberg, G. (1994). The incidence of back pain and headache among Swedish school children. Quality of Life Research, 3(1), S27-S31. https://doi.org/10.1007/BF00433372

Bronfman, N. C., \& López-Vázquez, E. L. (2011). A cross-cultural study of perceived benefit versus risk as mediators in the trustacceptance relationship. Risk Analysis: An International Journal, 31(12), 1919-1934. https://doi.org/10.1111/j.15396924.2011.01637.x

Carver, C. S., \& Scheier, M. F. (2014). Dispositional optimism. Trends in Cognitive Sciences, 18(6), 293-299. https://doi.org/10.1016/j.tics.2014.02.003

De la Cruz, M. V., Cordero, A., Seisdedos, N., \& González, M. (1988). Dominó 70: D-70. Adaptación española.

De Salud Pública, S. (2017). Encuesta nacional de salud 2016-2017 Primeros resultados. Santiago.

Del Barrio, V., Carrasco, M. Á., \& Holgado, F. P. (2006). Factor structure invariance in the Children's Big Five Questionnaire. European Journal of Psychological Assessment, 22(3), 158-167.

https://doi.org/10.1027/1015-5759.22.3.158 
Dingfelder, M. (2002). Cross secion calculations in condensed Media: Charged Particles in Liquid Water. Radiation Protection Dosimetry, 99(1-4), 23-27.

https://doi.org/10.1093/oxfordjournals.rpd.a00 6770

Durlak, J. A., \& Wells, A. M. (1997). Primary prevention mental health programs for children and adolescents: A metaanalytic review. American Journal of Community Psychology, 25(2), 115-152. https://doi.org/10.1023/A:1024654026646

Franko, D. L., Striegel-Moore, R. H., Thompson, D., Schreiber, G. B., \& Daniels, S. R. (2005). Does adolescent depression predict obesity in Black and White young adult women? Psychological Medicine, 35(10), 1505-1513. https://doi.org.10.1017/S0033291705005386

Greenberg, M. T., Weissberg, R. P., O'Brien, M. U., Zins, J. E., Fredericks, L., Resnik, H., \& Elias, M. J. (2003). Enhancing school-based prevention and youth development through coordinated social, emotional, and academic learning. American Psychologist, 58(6-7), 466-474. https://doi.org/10.1037/0003-066X.58.6-7.466

Hahn, R., Fuqua-Whitley, D., Wethington, H., Lowy, J., Crosby, A., Fullilove, M., ... \& Task Force on Community Preventive Services. (2007). Effectiveness of universal schoolbased programs to prevent violent and aggressive behavior: A systematic review. American Journal of Preventive Medicine, 33(2), S114-S129. https://doi.org/10.1016/j.amepre.2007.04.012

Hills, P., \& Argyle, M. (2002). The Oxford Happiness Questionnaire: A compact scale for the measurement of psychological well-being. Personality and individual differences, 33(7), 1073-1082.

https://doi.org/10.1016/S01918869(01)00213-6

Holden, E. W., Gladstein, J., Trulsen, M., \& Wall, B. (1994). Chronic daily headache in children and adolescents. Headache: The Journal of Head and Face Pain, 34(9), 508-514. https://doi.org/10.1111/j.1526-

4610.1994.hed3409508.x

Langeveld, J. H., Koot, H. M., Loonen, M. C. B., Hazebroek-Kampschreur, A. A. J. M., \&
Passchier, J. (1996). A quality of life instrument for adolescents with chronic headache. Cephalalgia, 16(3), 183-196. https://doi.org/10.1046/j.1468-

2982.1996.1603183.x

Langeveld, J. H., Koot, H., \& Passchier, J. (1999). Do experienced stress and trait negative affectivity moderate the relationship between headache and quality of life in adolescents? Journal of Pediatric Psychology, 24(1), 1-11.

Lira, C. J., Peinado, J. E., Solano-Pinto, N., Ornelas, M., \& Vega, H. B. (2020). Relaciones entre autoconcepto y bienestar psicológico en universitarias mexicanas. Revista Iberoamericana de Diagnóstico y Evaluación - e Avaliação Psicológica, 2(55), 59-70. https://doi.org/10.21865/RIDEP55.2.05 Londoño Pérez, C., Hernández Cortés, L. I. N. A., Alejo Castañeda, I. E., \& Pulido Garzón, D. (2013). Diseño y validación de la Escala de Optimismo Disposicional/Pesimismo-EOP. Universitas Psychologica, 12(1).

Marttila-Tornio, K., Ruotsalainen, H., Miettunen, J., Männikkö, N., \& Kääriäinen, M. (2020). Association between psychosocial problems and unhealthy health behavior patterns among Finnish adolescents. Child Psychiatry \& Human Development, 1-10. http://dx.doi.org/10.1007/s10578-020-00967-w Mohammadi, N., Aghayousefi, A., Nikrahan, G. R., Adams, C. N., Alipour, A., Sadeghi, M., ... \& Huffman, J. C. (2018). A randomized trial of an optimism training intervention in patients with heart disease. General Hospital Psychiatry, 51, 46-53.

https://doi.org/10.1016/j.genhosppsych.2017.1 2.004

Molinari, G., García-Palacios, A., Enrique, Á., Roca, P., Fernández-Llanio Comella, N., \& Botella, C. (2018). The power of visualization: Back to the future for pain management in fibromyalgia syndrome. Pain Medicine, 19(7), 1451-1468. https://doi.org/10.1093/pm/pnx298

Moutinho, H. A., Monteiro, A., Costa, A., \& Faria, L. (2019). Papel da inteligência emocional, felicidade e flow no desempenho académico e bem-estar subjetivo em contexto universitário. Revista Iberoamericana de Diagnóstico y Evaluación - e Avaliação 
Psicológica,

52(3),

99-114. https://doi.org/10.21865/RIDEP52.3.08.

Nolen-Hoeksema, S., Girgus, J. S., \& Seligman, M. E. (1992). Predictors and consequences of childhood depressive symptoms: A 5-year longitudinal study. Journal of Abnormal Psychology, 101(3), 405. https://doi.org/10.1037/0021-843X.101.3.405

Osborn, T. L., Rodriguez, M., Wasil, A. R., Venturo-Conerly, K. E., Gan, J., Alemu, R. G., ... \& Weisz, J. R. (2020). Single-session digital intervention for adolescent depression, anxiety, and well-being: Outcomes of a randomized controlled trial with Kenyan adolescents. Journal of consulting and clinical psychology, 88(7), 657. http://dx.doi.org/10.1037/ccp0000505

Payton, J., Weissberg, R. P., Durlak, J. A., Dymnicki, A. B., Taylor, R. D., Schellinger, K. B., \& Pachan, M. (2008). The Positive Impact of social and emotional learning for kindergarten to eighth-grade students: Findings from three scientific reviews. technical report. Collaborative for Academic, Social, and Emotional Learning (NJ1).

Peterson, C., \& Seligman, M.E.P. (1984). Causal explanations as a risk factor for depression: Theory and evidence. Psychological Review, 91(3), 347-374.

Peterson, C., Park, N., \& Seligman, M. E. (2005). Orientations to happiness and life satisfaction: The full life versus the empty life. Journal of Happiness Studies, 6(1), 25-41.

Pozar, F. (2002). Inventario de hábitos de estudio. Manual (9a. ed.). Madrid: Publicaciones de Psicología Aplicada, TEA Ediciones, S. A.

Quiceno, J. M., \& Vinaccia, S. (2014). Calidad de vida en adolescentes: Análisis desde las fortalezas personales $\mathrm{y}$ las emociones negativas. Terapia Psicológica, 32(3), 185200.

Remor, E., \& Amorós Gómez, M. (2012). Effect of an intervention program aimed at empowering strengths and psychological resources on mood, optimism, subjective health complaints and life satisfaction in university students. Acta Colombiana de Psicología, 15(2), 75-86.

Sandín, B., Chorot, P., Lostao, L., Joiner, T. E., Santed, M. A., \& Valiente, R. M. (1999).
Escalas PANAS de afecto positivo y negativo: Validación factorial y convergencia transcultural. Psicothema, 11(1), 37-51. https://www.redalyc.org/articulo.oa?id=727/7 2711104

Sanjuán, P. (2007). Post-performance attributions among depressive and no depressive subjects. Trabajo presentado en la 11th Biennial Meeting of the International Society for the Study of Individual Differences. Graz (Austria).

Scheier, M.F., \& Carver, C.S. (1985). Optimism, coping and health: Assessment and implications of generalized outcome expectancy. Health Psychology, 4(3), 219247.

https://doi.org/10.1037/0278-6133.4.3.219.

Seligman, M. E. P. (2011). Flourish: A visionary new understanding of happiness and wellbeing [Measurement instrument] New York.

Seligman, M. E. P., Kaslow, N. J., Alloy, L. B., Peterson, C., Tanenbaum, R. L., \& Abramson, L. Y. (1984). Attributional style and depressive symptoms among children. Journal of Abnormal Psychology, 93(2), 235238.

https://doi.org/10.1037/0021-843X.93.2.235.

Sherif, M. A., Cortes- Briones, J. A., Ranganathan, M., \& Skosnik, P. D. (2018). Cannabinoid-glutamate interactions and neural oscillations: Implications for psychosis. European Journal of Neuroscience, 48(8), 2890-2902.

http://dx.doi.org/10.1111/ejn.13800.

Steel, P., Schmidt, J., \& Shultz, J. (2008). Refining the relationship between personality and subjective well-being. Psychological Bulletin, 134(1), 138-161. https://doi.org/10.1037/0033-2909.134.1.138

Wilson, W. R. (1967). Correlates of avowed happiness. Psychological Bulletin, 67(4), 294. https://doi.org/10.1037/h0024431

Wong, D. F. K., Kwok, S. Y. C. L., Low, Y. T., Man, K. W., \& Ip, P. S. Y. (2018). Evaluating effectiveness of cognitive-behavior therapy for Hong kong adolescents with anxiety problems. Research on Social Work Practice, 28(5), 585-594.

http://dx.doi.org/10.1177/1049731516658351 